\title{
Improvement in Control Performance of a Servo System Compensating Bandwidth Variations at Low Speed
}

\author{
Young-Eun Ji*, Je-Wook Park**, Seon-Hwan Hwang**, Kwang-Ryul Baek ${ }^{* *}$, and Jang-Mok Kim ${ }^{\dagger}$ \\ * Dept. of Automation \& Control Research, Hyundai Heavy Industries, Ulsan, Korea \\ ${ }^{\dagger * *}$ School of Electrical Eng., Pusan National University, Busan, Korea
}

\begin{abstract}
This paper presents a novel design method for determining the PID gains of a speed controller for a servo system compensating variations in bandwidth at a low speed. The variations in bandwidth of a speed controller are measured at a low speed and the relationship between the bandwidth and the damping ratio are verified by determining the location of the closed loop pole. The proposed algorithm uses the z-transform of a plant and speed controller and applies the time-varying sampling method for determining the PID gains of the speed controller at low speed. The magnitude and the phase condition are considered for finding a suitable control gain. The usefulness and effectiveness of the proposed method is demonstrated through experimental results such as low speed control and robust disturbance responses.
\end{abstract}

Key Words: Bandwidth variation, Low speed, PID gains

\section{INTRODUCTION}

A PI (Proportional and Integral) controller is generally used as a method for speed control of motor drives in industrial servo systems. The $\mathrm{P}$ and I gains are determined by various factors such as the motor inertia, the torque constant and the bandwidth of the speed controller.

If the motor drive uses an incremental encoder as a position sensor and the rotor speed is calculated by the M/T method, the time delay in the speed control loop increases and the bandwidth of the speed controller decreases when the motor is controlled at a very low speed. This means the encoder pulse is detected less than 1 time per sampling period. The constant control gains in the low speed region decreases the control performance.

Conventional studies on the control of motors at low speed use an estimation of speed [1],[2], load torque and inertia [3] or a Kalman filter [4]. These methods focus on an additional algorithm for the improvement of control performance at low speed. However, these algorithms don't consider the performance improvement of the speed controller itself at low speed.

This paper proposes a novel method for determining the PID gains according to the variations in speed control bandwidth at low speed. The variations in speed control bandwidth at low speed are measured and the relation between the bandwidth

Manuscript received Sep. 24, 2009; revised May 7, 2010

$\dagger$ Corresponding Author: jmok@ pusan.ac.kr

Tel: +82-51-510-2366, Fax: +82-51-513-0212, Pusan Nat'l Univ.

* Automation \& Control Research, Hyundai Heavy Industries., Korea

** School of Electrical Eng., Pusan Nat'l Univ., Korea and the damping ratio is verified by determining the location of the closed pole.

The proposed algorithm uses a z-transform of the motor drive and applies the time-varying sampling method. The proper PID gains at low speed are determined by applying the magnitude and the phase condition in the z-domain. The experimental results verify the improved response and robustness of the proposed method at low speed.

\section{PID TUNING IN LOW SPEED COMPENSATION VARIATION OF CONTROL BANDWIDTH}

\section{A. Analysis of the time delay at low speed}

The M/T method is one of the most commonly used algorithms for speed measurement of servo motors [5]. The speed is calculated by counting the pulses of the incremental encoder and the internal timer of the microprocessor.

However, if the servo motor is controlled at a very low speed, no pulses from the encoder are injected to the controller during one sampling period. In this case, the time of the speed measurement is delayed and it decreases the bandwidth of the speed controller.

For example, assume that a 3600 pulse incremental encoder is used for a servo drive system and the sampling period of speed controller is $1[\mathrm{msec}]$. If the servo motor is controlled at less than $16[\mathrm{rpm}]$, no pulses are generated from the encoder during 1 cycle of the speed control.

In this case, the time delay of the speed measurement can be calculated using the internal counter of the microprocessor. 


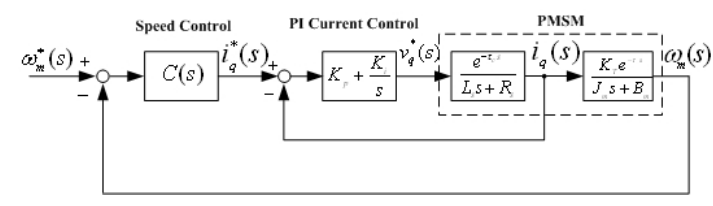

Fig. 1. Block diagram of the standard speed controller in s-domain.

The time delay of the speed measurement at low speed is shown in Table I.

TABLE I

TIME DELAY OF THE SPEED MEASUREMENT IN LOW SPEED

\begin{tabular}{|c|c|}
\hline $\begin{array}{c}\text { Speed }[\mathrm{rpm}] \\
14\end{array}$ & $\begin{array}{c}\text { Time delay }[\mathrm{\mu sec}] \\
205\end{array}$ \\
\hline 13 & 290 \\
\hline 12 & 405 \\
\hline 11 & 530 \\
\hline 10 & 680 \\
\hline 9 & 875 \\
\hline 8 & 1115 \\
\hline 7 & 1430 \\
\hline 6 & 1850 \\
\hline 5 & 2450 \\
\hline 4 & 3200 \\
\hline
\end{tabular}

B. Mathematical expression of the speed control loop of motor drives

Fig. 1 shows the block diagram of a standard speed controller and a PMSM (Permanent Magnet Synchronous Motor) in the s-domain [6]. The current controller generates the reference voltage of the motor and the speed controller outputs the current reference $i_{q}^{*}(s)$. The current controller can be simplified as a time delay function because the bandwidth of the current controller is much higher than the speed controller. $K_{p}$ and $K_{i}$ are the gains of the current controller. $\tau_{c}, L_{s}$ and $R$ are the time delay of current control loop, the inductance and the stator resistance, respectively. $J_{m}, B_{m}$ and $\tau s$ are the motor inertia, the friction and the time delay of the speed control loop, respectively.

The open-loop transfer function of the speed control loop in the s-domain is shown in (1).

$$
G_{p}(s)=\left(\frac{\omega_{c}}{s+\omega_{c}}\right)\left(\frac{K_{T}}{J_{m^{s}}+B_{m}}\right)
$$

where, $\tau_{c}$ is the bandwidth of the current controller and $K_{T}$ is the torque constant.

Fig. 2 shows the block diagram of a standard speed control loop in the z-domain. The open-loop transfer function of the speed control loop in the z-domain can be expressed as shown in (2).

$$
\begin{aligned}
& G_{p}(z)=z\left[\left(\frac{1-e^{-T s}}{s}\right) G_{p}(s)\right]=\left(1-z^{-1}\right) z\left(\frac{G_{p}(s)}{s}\right) \\
& =\frac{\left(\frac{b T}{a}-\frac{b}{a^{2}}+\frac{b T}{a^{2}} e^{-a T}\right) z+\left(\frac{b}{a}\right)\left(\frac{1}{a}-\frac{e^{-a T}}{a}-T e^{-a T}\right)}{z^{2}-\left(1+e^{-a T}\right) z+e^{-a T}} \\
& \left(a=\omega_{c}, b=\frac{K_{T \omega_{c}}}{J_{m}}\right)
\end{aligned}
$$

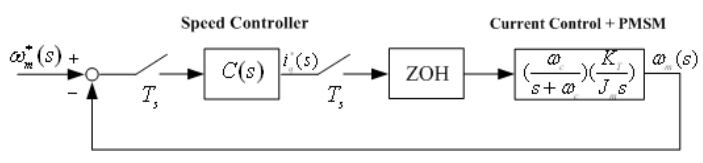

Fig. 2. Block diagram of approximated speed control loop of motor in z-domain.

where, $T$ is the sampling time of the speed controller.

Using the M/T method at low speed, the sampling period of the speed controller is varied according to the motor speed.

$$
\begin{aligned}
& G_{p}(z)=z\left[\left(\frac{1-e^{-T s}}{s}\right) G_{p}(s)\right] \\
& =\left(1-z^{-1}\right) z\left(\frac{G_{p}(s)}{s}\right) \\
& =\frac{\left(\frac{b T}{a}-\frac{b}{a^{2}}+\frac{b T}{a^{2}} e^{-a T}\right) z+\left(\frac{b}{a}\right)\left(\frac{1}{a}-\frac{e^{-a T}}{a}-T e^{-a T}\right)}{z^{2}-\left(1+e^{-a T}\right) z+e^{-a T}} .
\end{aligned}
$$

The PID controller can be expressed in the s-domain as shown in (4) and in the z-domain as shown in (5).

$$
G_{c}(z)=K_{s p}+K_{s d^{s}}+\frac{K_{s i}}{s}
$$

$G_{c}(z)=K_{s p}+\frac{K_{s d}(z-1)}{T z}+\frac{K_{s i} T(z+1)}{2(z-1)}$

$=\frac{\left(K_{s i} T^{2}+s K_{s p} T+2 K_{s d}\right) z^{2}+\left(K_{s i} T^{2}-2 K_{s p} T-4 K_{s d}\right) z+2 K_{s d}}{s T z(z-1)}$

The transfer function of the open loop control system is expressed as (6).

$$
G_{0}(z)=G_{p}(z) \times G_{c}(z) .
$$

After finding the transfer function, the location of the closed loop pole has to be determined. The closed loop pole in the s-domain is expressed as (7).

$$
\begin{aligned}
& s=-\zeta \omega_{n}+j \omega_{n} \sqrt{1-\zeta^{2}}=-\delta \omega_{n}+j \omega_{d} \\
& \left(\omega_{d}=\omega_{n} \sqrt{1-\delta^{2}}\right)
\end{aligned}
$$

where, $\xi$ is the damping ratio, $\omega_{n}$ is the natural frequency and $\omega_{d}$ is the damping frequency of oscillation.

In the z-domain, (7) is transformed as (8).

$$
\begin{aligned}
& z=e^{T s}=\exp \left(-\zeta \omega_{n} T+j \omega_{d} T\right) \\
& =\exp \left(-\frac{2 \pi \delta}{\sqrt{1-\delta^{2}}} \frac{\omega_{d}}{\omega_{s}}+j 2 \pi \frac{\omega_{d}}{\omega_{s}}\right) \quad\left(T=\frac{2 \pi}{\omega_{s}}\right) .
\end{aligned}
$$

Therefore, the location of the closed loop pole in the $\mathrm{z}$ domain can be expressed as shown in (9).

$$
|z|=\exp \left(-\frac{2 \pi}{\sqrt{1-\delta^{2}}} \frac{\omega_{d}}{\omega_{s}}\right), \angle z=2 \pi \frac{\omega_{d}}{\omega_{s}} .
$$

For determining $\omega_{d} / \omega_{s}$, the natural frequency $\omega_{n}$ has to be found. The natural frequency $\omega_{n}$ is selected to be $0.923[\mathrm{rad} / \mathrm{sec}]$ in this paper, because the PID gains are too large to control the servo motor if $\omega_{n}$ is larger than 0.923 . 


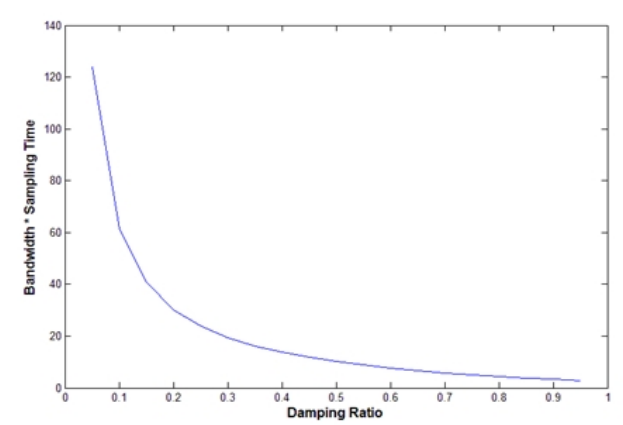

Fig. 3. The relation between $\omega_{B W} \times T_{s}$ and damping ratio $\xi$.

The bandwidth of the speed controller can be derived as the following.

The closed loop transfer function of the speed controller is shown in (10).

$$
\frac{G_{0}(s)}{1+G_{0}(s)}=\frac{\left(k_{p s}+\frac{k_{i s}}{s}+k_{d s^{s}}\right)\left(\frac{\omega_{c}}{s+\omega_{c}}\right)\left(\frac{K_{T}}{J_{m} s}\right)}{1+\left(k_{p s}+\frac{k_{i s}}{s}+k_{d s^{s}}\right)\left(\frac{\omega_{c}}{s+\omega_{c}}\right)\left(\frac{K_{T}}{J_{m} s}\right)}(10)
$$

By simplifying (10) as a second order transfer function, it can be expressed as (11).

$$
\frac{G_{0}(s)}{1+G_{0}(s)}=\frac{\omega_{n}^{2}}{s^{2}+\omega_{n} \zeta s+\omega_{n}^{2}} .
$$

Therefore, the amplitude of (11) at $\omega_{B W}$ is expressed as shown in (12).

$$
\begin{aligned}
& \left|\frac{\omega_{n}^{2}}{s^{2}+\omega_{n} \delta s+\omega_{n}^{2}}\right|_{\omega=\omega_{B W}} \\
& =\frac{\omega_{n}^{2}}{\sqrt{\left(\omega_{n}^{2}-\omega_{B W}\right)^{2}}+4 \delta^{2} \omega_{n}^{2} \omega_{B W}^{2}}=\frac{1}{\sqrt{2}}
\end{aligned}
$$

where, $\omega_{B W}$ is the bandwidth of the speed controller.

Therefore, the bandwidth of the speed controller can be calculated as (13),

$$
\omega_{B W}=\omega_{n} \sqrt{\left(1-2 \delta^{2}\right)+\sqrt{4 \delta^{4}-4 \delta^{2}+2}}
$$

and using the relation of (14),

$$
\omega_{n}=4 / T_{s} \delta .
$$

The relationship between the value of the bandwidth and the damping ratio can be expressed as (15).

$$
\omega_{B W} \times T_{s}=\frac{4}{\delta} \sqrt{\left(1-2 \delta^{2}\right)+\sqrt{4 \delta^{4}-4 \delta^{2}+2}} .
$$

If the sampling time is constant, the bandwidth of the speed controller decreases when the damping ratio grows as shown in Fig. 3. Therefore, if the motor is controlled at low speed, the bandwidth of the speed controller decreases and the damping ratio should be increased.

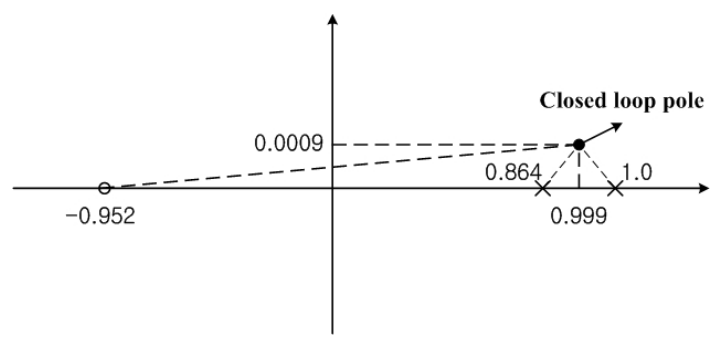

Fig. 4. The pole-zero plot of z-domain open-loop transfer function.

The bandwidth of the speed controller is determined by injecting a sinusoidal speed reference and increasing its frequency. When the phase of the speed response of the PMSM is delayed -45 degree, that point of frequency is the bandwidth of the speed controller. From (9) and (15), the location of the closed loop pole and the damping ratio can be determined as shown in Table II.

TABLE II

BANDWIDTH, DAMPING RATIO AND $\omega_{d} / \omega_{s}$ IN LOW SPEED

\begin{tabular}{|c|c|c|c|c|}
\hline Speed $[\mathrm{rpm}]$ & $\begin{array}{c}\text { Bandwidth } \\
{[\mathrm{rad} / \mathrm{sec}]}\end{array}$ & $\begin{array}{c}\text { Damping } \\
\text { ratio }\end{array}$ & $\omega_{d} / \omega_{s}$ & $\begin{array}{c}\text { Closed loop } \\
\text { pole }\end{array}$ \\
\hline 14 & 122.33 & 0.568 & $1 / 6892.18$ & $0.9994+i 0.00089$ \\
\hline 13 & 113.47 & 0.613 & $1 / 6678.38$ & $0.9993+i 0.00094$ \\
\hline 12 & 104.55 & 0.642 & $1 / 6131.73$ & $0.9991+i 0.00100$ \\
\hline 11 & 101.52 & 0.653 & $1 / 5874.09$ & $0.9991+i 0.00110$ \\
\hline 10 & 98.49 & 0.663 & $1 / 5412.05$ & $0.9990+i 0.00120$ \\
\hline 9 & 95.46 & 0.675 & $1 / 4917.15$ & $0.9988+i 0.00130$ \\
\hline 8 & 92.43 & 0.686 & $1 / 4423.12$ & $0.9987+i 0.00140$ \\
\hline 7 & 83.21 & 0.723 & $1 / 4054.56$ & $0.9984+i 0.00150$ \\
\hline 6 & 73.98 & 0.765 & $1 / 3708.35$ & $0.9980+i 0.00170$ \\
\hline 5 & 64.76 & 0.813 & $1 / 3384.34$ & $0.9974+i 0.00190$ \\
\hline 4 & 55.54 & 0.868 & $1 / 3236.68$ & $0.9966+i 0.00190$ \\
\hline
\end{tabular}

Assume, $T_{s}=0.0012[\mathrm{sec}], K_{T}=0.1132[\mathrm{Nm} / \mathrm{A}]$ and $J_{m}=0.0101\left[\mathrm{kgm}^{2}\right]$. From (6), the pole-zero plot of the zdomain open-loop transfer function is shown as in Fig. 4.

For determining the PID gains of a speed controller with a closed loop pole, two conditions should be considered. First, the phase of the closed loop pole should be -180 degrees.

From Fig. 4, the shortage angle from the view point of the plant and PID controller can be determined as (16).

$$
\begin{aligned}
& -0.0514^{\circ}-126.8956^{\circ}-126.8956^{\circ}-0.3783^{\circ}+ \\
& 0.0263^{\circ}+180^{\circ}=-74.195^{\circ} .
\end{aligned}
$$

This value should be compensated by two zeros of the PID controller. The shortage angle which should be compensated by each zero is $-74.195^{\circ} \div 2=-37.097^{\circ}$. The location of the zero of the PID controller can be determined to 0.9981 and the numerator of the transfer function of the PID controller is expressed as (17).

$$
(z-0.9981)(z-0.9981)=z^{2}-1.9962 z+0.9962 .
$$

Two polynomial expressions are obtained from (17). 


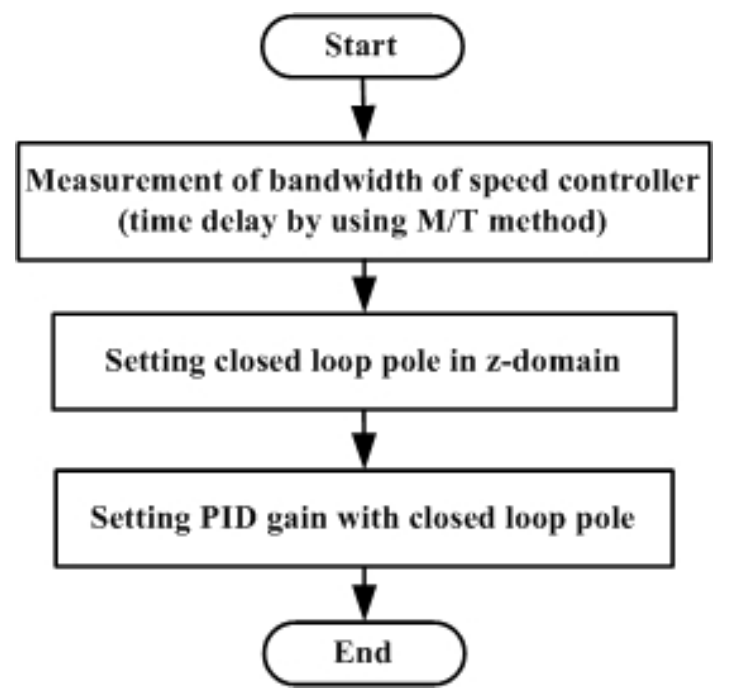

Fig. 5. Flowchart of the proposed algorithm.

$$
\begin{aligned}
& \frac{K_{s i} T^{2}-2 K_{s p} T-4 K_{s d}}{K_{s i} T^{2}+2 K_{s p} T+2 K_{s d}}=-1.9962 \\
& \frac{2 K_{s d}}{K_{s i} T^{2}+2 K_{s p} T+2 K_{s d}}=0.9962 .
\end{aligned}
$$

Second, by applying the magnitude condition that the magnitude of the closed loop pole has to be 1, the following polynomial is obtained.

$$
\begin{aligned}
& \left(K_{s i} T^{2}+2 K_{s p} T+2 K_{s d}\right) \\
& \left|\frac{(z-0.9981)^{2}}{2 T z(z-1)}\right|\left|G_{p}(z)\right|_{z=0.999+j 0.0009=1 .}
\end{aligned}
$$

The PID gains are determined from (18), (19) and (20) as shown in Table III.

TABLE III

CALCULATED VALUE OF PID GAINS IN LOW SPEED

\begin{tabular}{|c|c|c|c|}
\hline Speed $[\mathrm{rpm}]$ & $\mathrm{P}$ & $\mathrm{I}$ & $\mathrm{D}$ \\
\hline 14 & 0.1563 & 0.1217 & 0.0501 \\
\hline 13 & 0.1616 & 0.1216 & 0.0536 \\
\hline 12 & 0.1852 & 0.1372 & 0.0625 \\
\hline 11 & 0.1837 & 0.1324 & 0.0662 \\
\hline 10 & 0.1946 & 0.1355 & 0.0698 \\
\hline 9 & 0.2036 & 0.1393 & 0.0743 \\
\hline 8 & 0.2131 & 0.1433 & 0.0791 \\
\hline 7 & 0.2490 & 0.1590 & 0.0974 \\
\hline 6 & 0.3030 & 0.1828 & 0.1254 \\
\hline 5 & 0.3925 & 0.2230 & 0.1724 \\
\hline 4 & 0.5773 & 0.3071 & 0.2707 \\
\hline
\end{tabular}

The flow of the proposed algorithm is shown in Fig. 5.

\section{EXPERIMENTAL RESULTS}

The microprocessor of the servo drive system for the experiments is a TMS320VC33 (Texas Instruments) and the configuration of the servo system is shown in Fig. 6 and 7.

Fig. 8 shows the overall block diagram of the servo drive system.

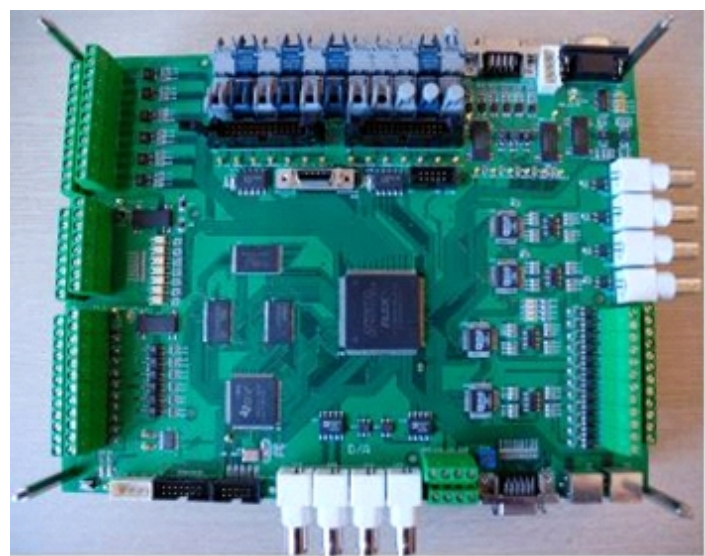

Fig. 6. Control board.

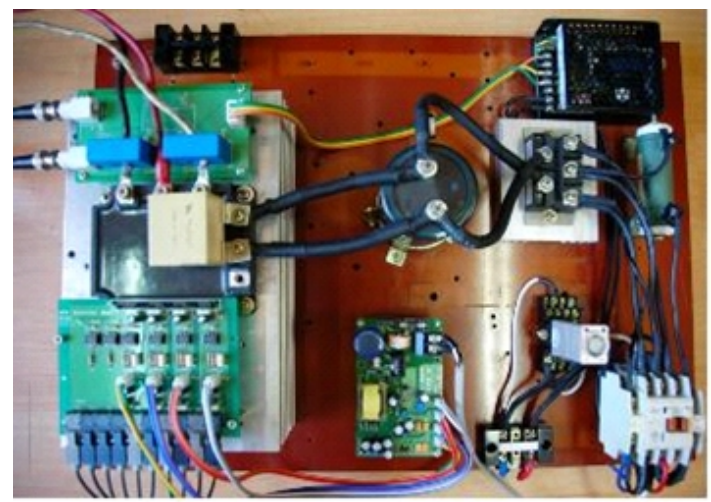

Fig. 7. Inverter board.

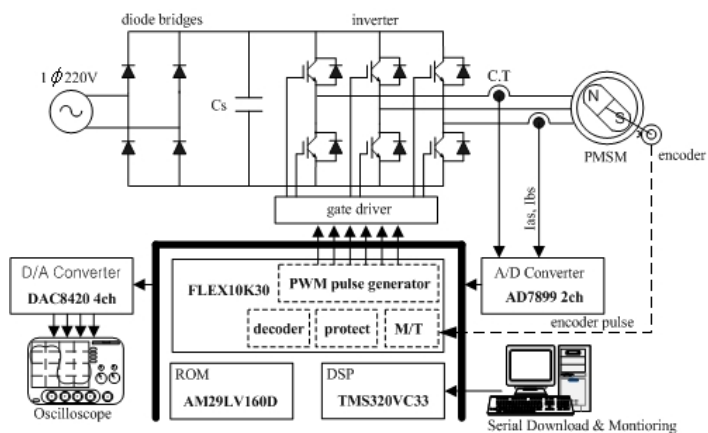

Fig. 8. Block diagram of the servo drive system.

The experiments are performed at low speed. The characteristics of the speed response and the disturbance response in the low speed range are shown in Fig. 9, 10, 11, and 12.

Fig. 9 and 10 show the speed response in the low speed range. Fig. 9 shows the speed response at a constant PID gains. If the speed reference is changed from $4[\mathrm{rpm}]$ to $7[\mathrm{rpm}]$ and the PID gains are constant, the waveform of its speed response contains ripple. On the other hand, as shown in Fig. 10, the speed response is improved and the speed ripple is reduced by compensating the bandwidth variation of the speed controller in the low speed region.

Fig. 11 shows the waveform of the disturbance response without compensation and Fig. 12 shows the disturbance response with the proposed algorithm in the low speed range. The servo motor is operating at $5[\mathrm{rpm}]$ and the external 


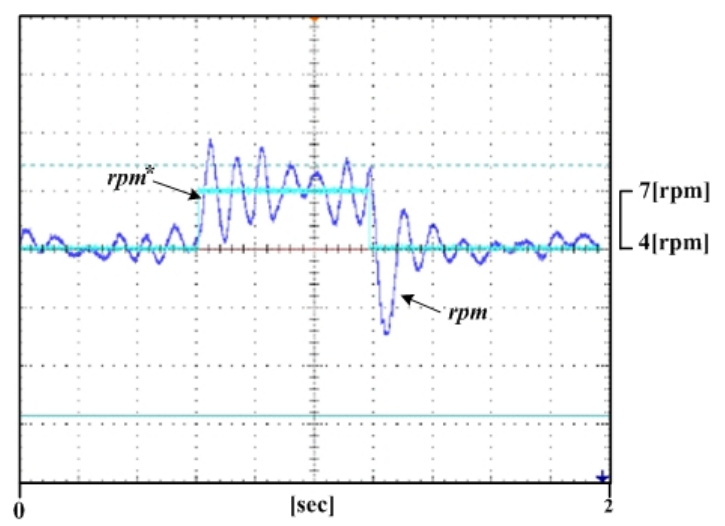

Fig. 9. Speed response of servo motor with constant PID gain.

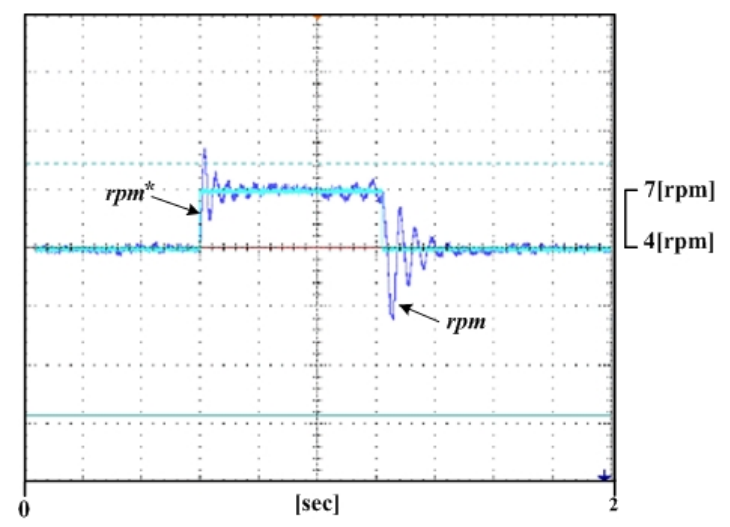

Fig. 10. Speed response of servo motor with compensating bandwidth variation.

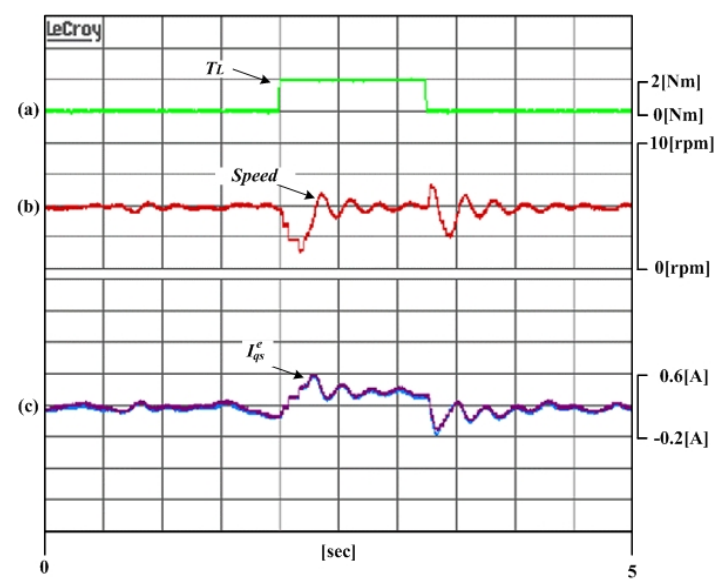

Fig. 11. Disturbance response when the PID gains are constant. (a) Load torque of external PMSM (b) Speed response of PMSM

(c)Q-axis current response of PMSM

disturbance is injected $2[\mathrm{Nm}]$ during $2.5[\mathrm{sec}]$ by the torque control of another coupled PMSM. The speed and q-axis current waveforms of Fig. 11 contain ripples in the transient state. Otherwise, the improved speed and q-axis current responses are obtained by implementing the proposed algorithm as shown in Fig. 12.

\section{CONCLUSIONS}

his paper suggests a novel PID tuning method considering the variation of the bandwidth of a speed controller in the low

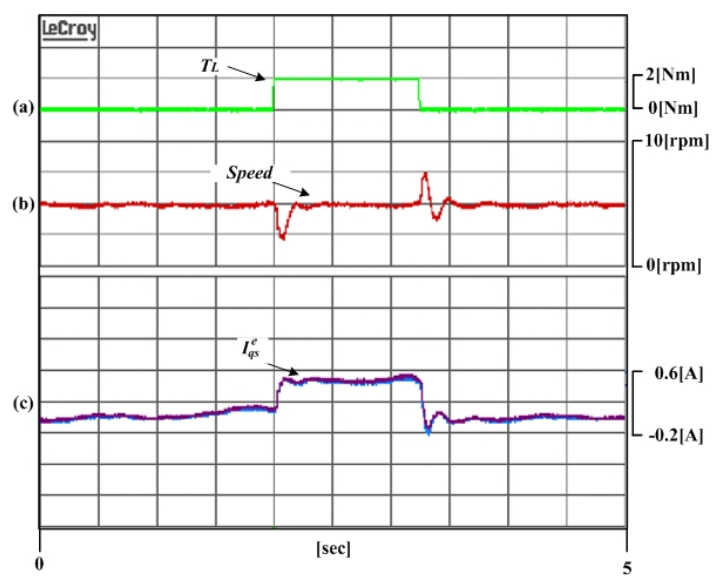

Fig. 12. Disturbance response with compensating bandwidth variation. (a) Load torque of external PMSM (b) Speed response of PMSM

(c) Q-axis current response of PMSM

speed range. The main contribution of this paper is the proof of the bandwidth variation of the PID gains in the low speed range. The experimental results verify the usefulness of the proposed algorithm.

The proposed algorithm can be easily implemented and requires less computation time.

\section{APPENDIX}

TABLE IV

PARAMETERS OF PMSM

\begin{tabular}{|c|c|c|c|}
\hline Rated voltage & $220[\mathrm{~V}]$ & Poles & 8 \\
\hline Rated current & $15.7[\mathrm{~A}]$ & Stator resistance & $0.0746[\Omega]$ \\
\hline Rated speed & $1800[\mathrm{rpm}]$ & Stator inductance & $1.144[\mathrm{mH}]$ \\
\hline Maximum speed & $2000[\mathrm{rpm}]$ & Back EMF constant & $0.092[\mathrm{~V} / \mathrm{rad} / \mathrm{s}]$ \\
\hline
\end{tabular}

\section{ACKNOWLEDGMENT}

This work was supported for two years by a Pusan National University Research Grant.

\section{REFERENCES}

[1] Gaolin Wang, "Low speed control of permanent magnet synchronous motor based on instantaneous speed estimation," Proceedings of the 6th world congress on intelligent control and automation, Vol.2, pp.80339036, 2006.

[2] Kung, Ying-Shieh, Huang, "High performance position controller for PMSM drives based on TMS320F2812 DSP," Proceedings of the IEEE International Conference on Control Applications, Vol. 1, pp. 290-295, 15-18 Aug., 2004.

[3] Kyo-Beum Lee, "Disturbance observer that uses radial basis function networks for the low speed control of a servo motor," IEE Proc.-Control Theory Appl., Vol.152, No.2, pp. 268-273, Mar. 2005.

[4] Heui-Wook Kim, Seung-Ki Sul, "A new motor speed estimator using kalman filter in low speed range," IEEE Trans. on Industrial Applications, Vol.43, No.4, pp.498-504, 1996.

[5] Yucheng Bai, Xiaogi Tang, Jihong Chen, Fangyu Peng, "Research on Ultra-low speed control of PMSM in servo system," Proceedings of the IEEE Intelligent Control and Automation, pp.2381-2386, 2008.

[6] Kichul Hong, "A Disturbance Torque Compensation Scheme Considering the Speed Measurement Delay," Proceedings of the IEEE Industry Applications Conference, Vol.1, pp.403-409, 1996. 


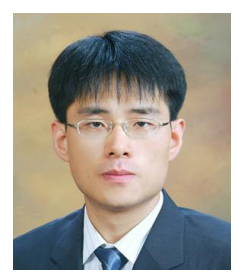

Young-Eun Ji was born in Busan, Korea, in 1980. He received his B.S. and M.S. in Electrical Engineering from Pusan National University, Busan, Korea, in 2006 and 2008, respectively. Since 2008, he has been with Hyundai Heavy Industries, Ulsan, Korea, where he is currently Researcher in the Automation \& Control Research Department. His research interests include digital control systems in heavy industries, automation of welding machines and the application of microcontrollers.

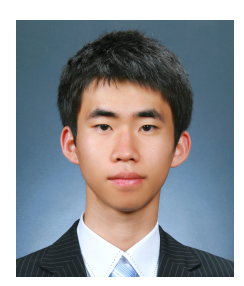

Je-Wook Park was born in Busan, Korea, in 1985. He received his B.S. and M.S. in Electrical Engineering from Pusan National University, Busan, Korea, in 2004 and 2006, respectively. He is currently working toward his Ph.D. at Pusan National University. His research interests include power conversion and electric machine drives.

Seon-Hwan Hwang was born in Chungnam, Korea, in 1978. He received his B.S. and M.S. in Electrical Engineering from Pusan National University, Busan, Korea, in 2004 and 2006, respectively. He is currently working toward his Ph.D. at Pusan National University. His research interests include power conversion and electric machine drives.

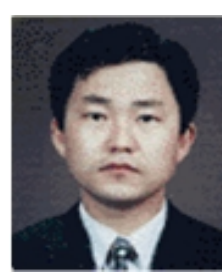

Kwang Ryul Baek received his B.S. in Electrical and Mechanical Engineering from Pusan National University in 1984, and his M.S and Ph.D. from KAIST, Daejeon, Korea, in 1986 and 1989, respectively. He joined the Turbotech Company as the Head of Development from 1989 to 1994. Now he is a Professor at Pusan National University. His research interests include digital signal processing, control systems, and highspeed circuit systems.

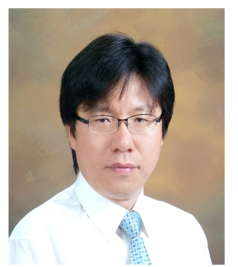

Jang-Mok Kim was born in Busan, Korea, in August 1961. He received his B.S. from Pusan National University in 1988, and his M.S. and Ph.D. from Seoul National University, Korea, in 1991 and 1996, respectively, in the department of Electrical Engineering. From 1997 to 2000, he was a Senior Research Engineer with the Korea Electrical Power Research Institute (KEPRI). Since 2001, he has been with the School of Electrical Engineering, Pusan National University (PNU), where he is currently a faculty member. In addition, he is a Research Member of the Research Institute of Computer Information and Communication at PNU. His present interests include the control of electric machines, electric vehicle propulsion, and power quality. 